\title{
ON THE SUMMABILITY OF A SEQUENCE OF WALSH FUNGTIONS
}

\author{
A. H. SIDDIQI
}

(Received 10 April 1968)

1.

The Rademacher functions are defined by

$$
\begin{aligned}
& \phi_{0}(x)=1\left(0 \leqq x<\frac{1}{2}\right), \quad \phi_{0}(x)=-1\left(\frac{1}{2} \leqq x<1\right), \\
& \phi_{0}(x+1)=\phi_{0}(x), \quad \phi_{n}(x)=\phi_{0}\left(2^{n} x\right), \quad(n=1,2,3, \cdots) \text {. }
\end{aligned}
$$

The Walsh functions are then given by

$$
\psi_{0}(x)=1, \quad \psi_{n}(x)=\phi_{n_{1}}(x) \cdot \phi_{n_{2}}(x) \cdots \phi_{n_{r}}(x)
$$

for $n=2^{n_{1}}+2^{n_{2}}+2^{n_{3}}+\cdots+2^{n_{r}}$, where the integers $n_{i}$ are uniquely determined by $n_{i+1}<n_{i}$.

Let $f(x)$ be an integrable function in the sense of Lebesgue in $[0,1]$ and be periodic with period 1. Let the Walsh-Fourier series of $f(x)$ be $\sum_{n=1}^{\infty} a_{n} \psi_{n}(x)$, where

$$
a_{n}=\int_{0}^{1} f(x) \psi_{n}(x) d x .
$$

We shall now enumerate important properties and results concerning Walsh-Functions which have been obtained by Fine [3] and which have played a significant role in the theory of Walsh-Fourier series.

The dyadic group $G$ may be defined as the countable direct product of the groups with elements 0 and 1 , in which the group operation is addition modulo 2. Thus the dyadic group $G$ is the set of all 0,1 sequences in which the group operation, which we shall denote by $\dot{+}$, is addition modulo 2 for each element.

Let $\bar{x}$ be an element of $G, \bar{x}=\left\{x_{1}, x_{2}, \cdots\right\}, x_{n}=0,1$. We define the function

$$
\lambda(\bar{x})=\sum_{n=1}^{\infty} 2^{-n} x_{n}
$$

The function $\lambda$, which maps $G$ onto the closed interval $[0,1]$, does not have a single-valued inverse on the dyadic rationals. We shall agree to take the finite expansion in that case. Thus for all real $x$, if we write the inverse as $\mu$, 


$$
\lambda(\mu(x))=x-[x] .
$$

If $\bar{x}=\left\{x_{n}\right\}$ and $\bar{y}=\left\{y_{n}\right\}$ are elements of $G$, we have

$$
\bar{x} \dot{+} \bar{y}=\left\{\left|x_{n}-y_{n}\right|\right\} .
$$

We shall abbreviate $\lambda(\mu(x) \dot{+} \mu(y))$ as $x \dot{+} y$ for any real $x$ and $y$. Then, if

$$
x=\sum_{n=1}^{\infty} 2^{-n} x_{n}, \quad y=\sum_{n=1}^{\infty} 2^{-n} y_{n}
$$

$x_{n}$ and $y_{n}=0,1$, we have by $(1.2)$ and (1.3)

$$
x \dot{+} y=\sum_{n=1}^{\infty} 2^{-n}\left|x_{n}-y_{n}\right| \text {. }
$$

For any real number $x$ and $h$, we have

$$
|(x+h)-(x-[x])| \leqq h-[h] .
$$

In particular if $0 \leqq x<1,0 \leqq h<1$, then we have

$$
|(x \dot{+} h)-x| \leqq h \text {. }
$$

For each fixed $x$ and for almost all $t$, the equation

$$
\psi_{n}(x \dot{+} t)=\psi_{n}(x) \psi_{n}(t) \text { holds. }
$$

Also for each fixed $x$

$$
\int_{0}^{1} f(x \dot{+} t) d t=\int_{0}^{1} f(t) d t
$$

and

$$
\int_{0}^{1} f(t) \psi_{n}(x \dot{+} t) d t=\int_{0}^{1} f(x \dot{+} t) \psi_{n}(t) d t
$$

Let

$$
\begin{aligned}
& J_{k}(y)=\int_{0}^{y} \psi_{k}(t) d t, \\
& J_{k}^{*}(y)=k J_{k}(y) .
\end{aligned}
$$

For $k \geqq 1$, we write $k=2^{n}+k^{\prime}$, where $0 \leqq k^{\prime}<2^{n}, n=0,1,2, \cdots$. We have also

$$
J_{k}(y)=2^{-(n+2)}\left\{\psi_{k^{\prime}}(y)-\sum_{r=1}^{\infty} 2^{-r} \psi_{2^{n+r}+k}(y)\right\} .
$$

It is easy to see that

$$
2^{n+2} J_{k}(y)=0, \quad \text { for } y=0,1
$$

and

$$
\left|J_{k}^{*}(y)\right| \leqq M \quad \text { for all } y \text { and } k
$$


Let $B_{k}(x)$ denote the sequence $\left\{k a_{k} \psi_{k}(x)\right\}$, where $a_{k}$ is Walsh-Fourier coefficient of a function of bounded variation.

Let $A=\left(a_{m, k}\right)$ be an infinite matrix of real or complex numbers and $\left\{s_{k}\right\}$ be any sequence of real numbers. With every sequence $\left\{s_{k}\right\}$ we associate a sequence $\left\{\sigma_{n}\right\}$ given by

$$
\sigma_{m}=\sum_{k=0}^{\infty} a_{m, k} s_{k},
$$

provided the series on the right converges for all $m$. The sequence $\left\{\sigma_{m}\right\}$ is called the $A$-transform of $\left\{s_{k}\right\}$.

If $\sigma_{m} \rightarrow s$ as $m \rightarrow \infty$, we say that the sequence $\left\{s_{k}\right\}$ is $A$-summable to $s$. The matrix $A$ is called regular if it satisfies the following conditions:

(i) $\lim _{m \rightarrow \infty} a_{m, k}=0$ for $k=0,1,2,3, \cdots$

(ii) $\sup _{m} \sum_{k=0}^{\infty}\left|a_{m, k}\right| \leqq M$,

(iii) $\lim _{m \rightarrow \infty} \sum_{k=0}^{\infty} a_{m, k}=1$.

The matrix $A$ is called triangular, if $a_{m, k}=0$ for $k>m$.

We say that a bounded sequence $\left\{s_{k}\right\}$ is almost convergent [4] to the sum $l$ if

$$
\lim _{p \rightarrow \infty} \frac{1}{p} \sum_{k=0}^{p-1} s_{n+k}=l
$$

uniformly in $n$. Every almost convergent sequence is summable $(C, \alpha)$, $\alpha>0$ [4] and the limits are equal.

A sequence $\left\{s_{k}\right\}$ is said to be almost $A$-summable [2] to $s$ if the $A$ transform of $\left\{s_{k}\right\}$ is almost convergent to $s$ and the matrix $A$ is said to be almost regular if $s_{k} \rightarrow s$ implies that $\left\{\sigma_{n}\right\}$ is almost convergent to $s$. The necessary and sufficient conditions for the matrix $A$ to be almost regular [2] are:

(a) $\quad \sup _{m} \sum_{k=0}^{\infty}\left|a_{m, k}\right|<M_{1}, m=+1,+2,+3, \cdots$

where $M_{1}$ is a positive constant.

(b) $\lim _{p \rightarrow \infty} \frac{1}{p} \sum_{j=n}^{n+p-1} a_{j, k}=0$ uniformly in $n, k=0,1,2, \cdots$

(c) $\quad \lim _{p \rightarrow \infty} \frac{1}{p} \sum_{j=n}^{n+p-1} \sum_{k=0}^{\infty} a_{j, k}=1$, uniformly in $n$.

A sequence $\left\{s_{k}\right\}$ is said to be $F A$-summable [4] to the limit $s$ if 


$$
\sigma_{m, k}^{*}=\sum_{q=0}^{\infty} a_{m, q} s_{q+k}
$$

tends to $s$ as $m \rightarrow \infty$, uniformly in $k$.

It is known [4] that every $F A$-summable sequence is almost convergent if $A$ is a regular matrix.

A sequence $\left\{s_{k}\right\}$ will be said to be $A B$-summable to the limit $s$ if its $A$-transform is $F B$-summable to the limit $s$ where $B=\left(b_{m, k}\right)$ is an infinite matrix.

It is easy to see that every $A B$-summable sequence is almost $A$ summable provided the second matrix $B$ is regular.

\section{2.}

In 1947, Fine [3] proved the following theorems concerning WalshFourier coefficients of function of bounded variation and absolutely continuous function.

THEOREM A. If $f(x)$ is of bounded variation, and $V$ is its total variation over $(0,1)$, then

$$
\left|u_{k}\right| \leqq V \mid k, \text { for } k>0 \text {. }
$$

THEOREM B. The only absolutely continuous functions whose Fourier coefficients satisfy $a_{k}=0(1 / k)$ are the constants.

This result shows a marked difference in the behaviour of WalshFourier series and ordinary Fourier series of absolutely continuous functions. Morgenthaler [5] proved a theorem which shows that 'on the average' the coefficients behave as they do in the classical system.

His result is as follows:

Theorem C. Let $f(x)$ be real valued, periodic, and of mean value zero on $[0,1] . I f$

$$
F(x)=\int_{0}^{x} f(t) d t \text { and } F(x) \sim \sum_{k=0}^{\infty} b_{k} \psi_{k}(x),
$$

then the arithmetic means of the sequence $k\left|b_{k}\right|$ tend to zero.

In the present paper we shall obtain necessary and sufficient conditions in order that the sequence $\left\{B_{k}(x)\right\}$ be $(A)$, almost $A, F A$ and $A B$-summable.

We shall also deduce an interesting corollary concerning WalshFourier coefficients of functions of bounded variation.

3.

In what follows we shall prove the following theorems:

THEOREM 1. If $(A)$ is regular, then for every $f \in B V[0,1]$ and for every $x \in[0,1]$ 


$$
\lim _{m \rightarrow \infty} \sum_{k=0}^{m} a_{m, k} B_{k}(x)=0
$$

if and only if

$$
\lim _{m \rightarrow \infty} \sum_{k=0}^{m} a_{m, k} J_{k}^{*}(t)=0
$$

in every $0<\delta<t \leqq 1$, where $(A)$ is a triangular matrix and $\delta$ is small.

THEOREm 2. If $A$ be almost regular, then for every $f \in B V[0,1]$ and for every $x \in[0,1]$

$$
\lim _{p \rightarrow \infty} \frac{1}{p} \sum_{r=0}^{p-1} \sum_{k=0}^{\infty} a_{m+r}, k B_{k}(x)=0
$$

uniformly in $m$, if and only if

$$
\lim _{p \rightarrow \infty} \sum_{r=0}^{p-1} \sum_{k=0}^{\infty} a_{m+r}, k J_{k}^{*}(t)=0
$$

uniformly in $m$ for every $0<\delta \leqq t \leqq 1, \delta$ is small.

TheORem 3. If $A=\left(a_{m, k}\right)$ is regular, then for every $f \in B V[0,1]$ and for every $x \in[0,1]$, the sequence $\left\{B_{k}(x)\right\}$ is $F A$-summable to the limit zero if and only if

$$
\lim _{m \rightarrow \infty} \sum_{v=k}^{\infty} a_{m, v-k} J_{v}^{*}(t)=0,
$$

uniformly in $k$ in the interval $0<\delta \leqq t \leqq 1$ where $\delta$ is small.

THEOREM 4. If $\left(a_{m, k}\right)$ and $\left(b_{m, k}\right)$ be two infinite matrices satisfying the condition:

$$
\sup _{m} \sum_{v=k}^{\infty}\left|b_{m, v-k}\right| \sum_{j=0}^{\infty}\left|\boldsymbol{a}_{v, j}\right|<\infty
$$

uniformly in $k$, then for every $f \in B V[0,1]$ and for every $x \in[0,1]$ the sequence $\left\{B_{k}(x)\right\}$ is $A B$-summable to the limit zero, if and only if

$$
\lim _{m \rightarrow \infty} \sum_{j=k^{\prime}}^{\infty} b_{m, j-k^{\prime}} \sum_{k=0}^{\infty} a_{j, k} J_{k}^{*}(t)=0
$$

uniformly in $k^{\prime}$ in every interval $0<\delta \leqq t \leqq 1, \delta$ is small.

4.

Proof of Theorem 1 . We have by virtue of (1.7) and (1.9) 


$$
\begin{aligned}
\sum_{k=0}^{m} a_{m, k} B_{k}(x) & =\sum_{k=0}^{m} a_{m, k} k a_{k} \psi_{k}(x) \\
& =\sum_{k=0}^{m} a_{m, k} k \int_{0}^{1} f(t) \psi_{k}(t) \psi_{k}(x) d t \\
& =\sum_{k=0}^{m} a_{m, k} k \int_{0}^{1} f(t) \psi_{k}(x \dot{+} t) d t \\
& =\sum_{k=0}^{m} a_{m, k} k \int_{0}^{1} f(x \dot{+} t) \psi_{k}(t) d t \\
& =\sum_{k=0}^{m} a_{m, k} k\left[f(x \dot{+} t) J_{k}(t)\right]_{0}^{1}-\sum_{k=0}^{m} a_{m, k} k \int_{0}^{1} J_{k}(t) d f(x \dot{+} t) \\
& =0-\int_{0}^{1} \sum_{k=0}^{m} a_{m, k} J_{k}^{*}(t) d f(x \dot{+} t) .
\end{aligned}
$$

Let

$$
K_{m}(t)=\sum_{k=0}^{m} a_{m, k} J_{k}^{*}(t) .
$$

We have to show that if (3.1) holds, then for every $f \in B V[0,1]$ and for every $x \in[0,1]$,

$$
\lim _{m \rightarrow \infty} \int_{0}^{1} K_{m}(t) d \Phi_{x}(t)=0, \quad \text { where } \Phi_{x}(t)=f(x+t),
$$

and conversely.

Condition (4.1) is equivalent to following condition

$$
\lim _{m \rightarrow \infty} \int_{\delta}^{1} K_{m}(t) d \Phi_{x}(t)=0
$$

for every $f \in B V[0,1]$ and for every $x \in[0,1]$ and for $0<\delta<1$.

For if $f \in B V[0,1]$ and $x \in[0,1]$, given any $\varepsilon>0$, there exists a $\delta>0$ such that

$$
\int_{0}^{\delta}\left|d \Phi_{x}(t)\right|<\frac{\varepsilon}{2 M} .
$$

By virtue of the regularity condition we have

$$
\left|K_{m}(t)\right| \leqq \sum_{k=0}^{m}\left|a_{m, k}\right|\left|J_{k}^{*}(t)\right| \leqq M \sum_{k=0}^{m}\left|a_{m, k}\right| \leqq M
$$

so that

$$
\left|\int_{0}^{1} K_{m}(t) d \Phi_{x}(t)-\int_{\delta}^{1} K_{m}(t) d \Phi_{x}(t)\right|=\left|\int_{0}^{\delta} K_{m}(t) d \Phi_{x}(t)\right| \mid<\frac{\varepsilon}{2} .
$$

Thus conditions (4.1) and (4.2) are equivalent. 
By a theorem on Weak convergence $[1, p .134]$ of sequences in the Banach space of all continuous functions defined in a finite closed interval it follows that (4.2) holds if and only if

(i) $\left|K_{m}(t)\right| \leqq M$ for all $m$ and $t$ in $[\delta, 1]$ and

(ii)' (3.1) holds.

Since (i)' always holds by virtue of (4.4), it follows that (4.2) holds if and only if (3.1) holds.

This completes the proof of Theorem 1.

5.

Proof of Theorem 2. We have as in the proof of the previous theorem,

$$
\begin{aligned}
& \frac{1}{p} \sum_{r=0}^{p-1} \sum_{k=0}^{\infty} a_{m+r, k} B_{k}(x) \\
&=\frac{1}{p} \sum_{r=0}^{p-1} \sum_{k=0}^{\infty} a_{m+r, k} k \int_{0}^{1} f(t \dot{+} x) \psi_{k}(t) d t \\
&=\frac{1}{p} \sum_{r=0}^{p-1} \sum_{k=0}^{\infty} a_{m+r, k} k\left[f(x \dot{+} t) J_{k}(t)\right]_{0}^{1} \\
&-\frac{1}{p} \sum_{r=0}^{p-1} \sum_{k=0}^{\infty} a_{m+r, k} \int_{0}^{1} d f(x \dot{+} t) J_{k}^{*}(t) \\
&= I_{1}-I_{2}, \text { say. }
\end{aligned}
$$

Since $I_{1}=0$, it is sufficient to show that

$$
I_{2}=\int_{0}^{1} d f(x+t) K_{m, p}(t) \rightarrow 0, \quad \text { as } p \rightarrow \infty
$$

uniformly in $m$, where

$$
K_{m, p}(t)=\frac{1}{p} \sum_{r=0}^{p-1} \sum_{k=0}^{\infty} a_{m+r, k} J_{k}^{*}(t)
$$

By virtue of the condition (a) and (1.12) we have

$$
\left|K_{m, p}(t)\right| \leqq M_{2}
$$

uniformly in $m$ and therefore we can show, as in the proof of theorem 1 that condition (5.1) is equivalent to the following condition:

$$
\int_{\delta}^{1} K_{m, p}(t) d f(x \dot{+} t) \rightarrow 0 \text { as } p \rightarrow \infty
$$

uniformly in $m$.

Following the lines of Banach $[1$, p. 134] it can be easily verified that a 
sequence of continuous functions $\left\{x_{n}^{k}(t)\right\}$ converges weakly (uniformly in $k$ ) to a continuous function $x(t)$ that is

$$
\lim _{n \rightarrow \infty} \int_{0}^{1} x_{n}^{k}(t) d g(t)=\int_{0}^{1} x(t) d g(t)
$$

uniformly in $k$ for every $g \in B V$, if and only if

(i) $x_{n}^{k}(t)$ is bounded uniformly in $k, n=1,2, \cdots$.

(ii) $\lim _{n \rightarrow \infty} x_{n}^{k}(t)=x(t)$ uniformly in $k=0,1, \cdots$ for every $t \in[0,1]$.

Applying this theorem and the fact that $\left|K_{m, p}(t)\right| \leqq M_{2}$ for all $m, p$ and $t \in[\delta, \mathbf{1}]$, the theorem follows.

6.

Proof of Theorem 3 . We have by virtue of (1.7) and (1.9)

$$
\begin{aligned}
\sigma_{m, k}^{*}= & \sum_{v=0}^{\infty} a_{m, v} s_{v+k} \\
= & \sum_{j=k}^{\infty} a_{m, j-k} s_{j} \\
= & \sum_{j=k}^{\infty} a_{m, j-k} j a_{j} \psi_{j}(x) \\
= & \sum_{j=k}^{\infty} a_{m, j-k}\left(j \int_{0}^{1} f(t) \psi_{j}(t) d t\right) \psi_{j}(x) \\
= & \sum_{j=k}^{\infty} a_{m, j-k} j \int_{0}^{1} f(x \dot{+} t) \psi_{j}(t) d t \\
= & \sum_{j=k}^{\infty} a_{m, j-k} j\left[f(x \dot{+} t) J_{j}(t)\right]_{0}^{1} \\
& -\sum_{j=k}^{\infty} a_{m, j-k} j\left(\int_{0}^{1} d(f(x \dot{+} t)) J_{j}(t)\right) \\
= & \sum_{j=k}^{\infty} a_{m, j-k}\left[J_{j}^{*}(t) f(x \dot{+} t)\right]_{0}^{1} \\
& -\sum_{j=k}^{\infty} a_{m, j-k} \int_{0}^{1} d\left(f(x \dot{+} t) J_{j}^{*}(t)\right. \\
= & L_{1}-L_{2}, \operatorname{say}
\end{aligned}
$$

$L_{1}=0$ uniformly in $k$.

In order to prove the theorem we have to show that if $(3.3)$ holds, then for every $f \in B V[0,1]$ and for every $x \in[0,1]$ 


$$
\lim _{m \rightarrow \infty} \int_{0}^{1} K_{m, k}^{*}(t) d f(x \dot{+} t)=0
$$

uniformly in $k$, where

$$
K_{m, k}^{*}(t)=\sum_{j=k}^{\infty} a_{m, j-k} J_{j}^{*}(t)
$$

and conversely.

Proceeding on the lines of the proof of theorem 2 we can show that condition $(6.1)$ is equivalent to

$$
\lim _{m \rightarrow \infty} \int_{0}^{1} K_{m, k}^{*}(t) d(f(x+t)=0
$$

uniformly in $k$, for every $f \in B V[0,1]$, for every $x \in[0,1]$ and for $0<\delta<1$. Thus it follows as shown in Theorem 2, that (6.2) holds, if and only if

$\left(\mathrm{a}^{\prime}\right)\left|K_{m, k}^{*}(t)\right| \leqq M, m=1,2, \cdots$ and $t \in[\delta, 1]$ for $\delta>0$ and uniformly in $k$.

(b') (3.3) holds.

Since (a)' always holds, it follows that (6.2) holds if and only if (3.3) holds.

This completes the proof of Theorem 3 .

\section{7.}

Proof of Theorem 4. We have

so that

$$
\sigma_{m}=\sum_{k=0}^{\infty} a_{m, k} k a_{k} \psi_{k}(x)
$$

$$
\begin{aligned}
\sigma_{m, k^{\prime}}^{*}= & \sum_{v=\mathbf{0}}^{\infty} b_{m, v} \sigma_{v+k^{\prime}} \\
= & \sum_{v=0}^{\infty} b_{m, v} \sum_{k=0}^{\infty} a_{v+k^{\prime}} k a_{k} \psi_{k}(x) \\
= & \sum_{j=k^{\prime}}^{\infty} b_{m, j-k^{\prime}} \sum_{k=0}^{\infty} a_{j k} k \int_{0}^{1} f(x \dot{+} t) \psi_{k}(t) d t \\
= & \sum_{j=k^{\prime}}^{\infty} b_{m, j-k^{\prime}} \sum_{k=0}^{\infty} a_{j, k}\left[J_{k}^{*}(t) f(x \dot{+} t)\right]_{0}^{1} \\
& -\sum_{j=k^{\prime}}^{\infty} b_{m, j-k^{\prime}} \sum_{k=0}^{\infty} a_{j, k} \int_{0}^{1} d\left(f(x \dot{+} t) J_{k}^{*}(t)\right. \\
= & N_{\mathbf{1}}-N_{2}, \text { say. }
\end{aligned}
$$

But $N_{1}=0$. 
Proceeding on the lines of the proof of Theorem 3 we can show that $N_{2}=0$, as $m \rightarrow \infty$, uniformly in $k^{\prime}$ if and only if

$$
\lim _{m \rightarrow \infty} \sum_{j=k^{\prime}}^{\infty} b_{m, j} j_{j-k^{\prime}}^{\infty} \sum_{k=0}^{\infty} a_{j, k} J_{k}^{*}(t)=0
$$

uniformly in $k^{\prime}$ in the interval $0<\delta \leqq t \leqq 1$.

This completes the proof of the theorem 4 .

8.

$$
\text { If we take } \quad \begin{array}{rlrl}
x=0, k=0, a_{n, \nu} & =\frac{1}{n}, & v<n \\
& =0 & v \geqq n,
\end{array}
$$

we get the following corollary of theorem 3 which bridges the gap between theorems $A$ and $C$.

Corollary. If $f \in B V[0,1]$, then $\left\{k a_{k}\right\}$ is summable $(C, 1)$ to zero if and only if

$$
\lim _{m \rightarrow \infty} \frac{1}{m} \sum_{v=0}^{m-1} J_{v}^{*}(t)=0
$$

for $0<\delta \leqq t \leqq 1$

The author would like to express his thanks to Prof. J. A. Siddiqi for his encouragement in the preparation of this paper.

\section{References}

[1] S. Banach, Théorie des opérations linéaires (Warszawa, 1932)

[2] J. P. King, 'Almost convergent sequences', Proc. Amer., Math. Soc. 17 (1966), 1219-1225.

[3] N. J. Fine, 'On the Walsh-functions', Trans. Amer. Math. Soc. 65 (1949), 372-414.

[4] G. G. Lorentz, 'A contribution to the theory of divergent sequences', Acta. Math. 80 (1948), 167-190.

[5] G. W. Morgenthaler, 'On Walsh-Fourier series', Trans. Amer. Math. Soc. 84 (1957 $472-507$.

Aligarh Muslim University

Aligarh, U.P.

India 